\title{
Retention following appetitive discrimination training: The Kamin effect
}

\author{
JEFFREY A. SEYBERT, LINDA G. McCLANAHAN, and J. WESLEY GILLILAND \\ University of Missouri, Kansas City, Missouri 64110
}

\begin{abstract}
Two experiments were performed to investigate retention of a discriminative response under conditions of appetitive motivation. In Experiment 1, four groups of rats received 20 training trials in a two-choice position discrimination procedure. Following training, the subjects received 16 test trials $.05,1$, or $24 \mathrm{~h}$ later on a reversal of the original discrimination problem. The results revealed that retention of the original discriminative training was a U-shaped function of time following training (i.e., a Kamin effect was observed), in terms of both associative (percent errors) and performance (response latencies) measures. The data from the second experiment ruled out certain nonassociative explanations for the results of Experiment 1. Thus, the results of these experiments extend the observation of nonmonotonic retention functions to appetitive discrimination learning and provide further support for the hypothesis that an associative mechanism is responsible for such functions.
\end{abstract}

The Kamin effect (a nonmonotonic, U-shaped retention function; Kamin, 1957) has been demonstrated in a wide variety of experimental paradigms under conditions of both aversive and appetitive motivation. Two hypotheses have been suggested as explanations of the Kamin effect. The nonassociative hypothesis (e.g., Pinel \& Mucha, 1973) states that retention performance is some function of the animal's level of motivation or activity as a result of exposure to an aversive stimulus (like shock). The associative hypothesis, on the other hand, attributes Kamin effects to associative or learning factors, rather than to motivation or activity (e.g., Bryan \& Spear, 1976). Both the associative and nonassociative hypotheses have received considerable empirical support (cf. Anisman, 1975; Bryan \& Spear, 1976).

It has been suggested elsewhere (Kamin, 1957; Seybert, Vandenberg, Harvey, Budd, \& McClanahan, 1979) that investigation of retention of appetitive responses might provide more definitive information regarding this associative/nonassociative controversy. Since the nonassociative hypotheses all rely on the effects of experience with an aversive stimulus (like shock), they encounter serious difficulty in explaining Kamin effects in situations in which no such stimulus is present (e.g., appetitive learning situations). Although the Kamin effect has been demonstrated in a simple, nondifferential appetitive learning situation (Seybert et al., 1979), examination of retention in an appetitive discrimination paradigm might also prove very helpful in this regard. However, the results of the two studies that have examined retention of an appetitive discrimi-

Requests for reprints should be sent to J. A. Seybert, whose current address is: Office of Institutional Research, Johnson County Community College, College Blvd. at Quivira, Overland Park, Kansas 66210. nation (Jaffard, Destrade, Soumireu-Mourat, \& Cardo, 1974; Tribhowan, Rucker, \& McDiarmid, 1971) are inconclusive because they used food-deprivation procedures. Thus, the potential advantage of using an appetitive paradigm to evaluate retention of an associative effect may have been lost due to a nonassociative confound (differential motivation). The present experiments were designed to investigate retention of appetitive discrimination learning using a procedure that obviated any possible confound due to differential motivation.

\section{EXPERIMENT 1}

\section{Method}

Subjects. Thirty-two experimentally naive male albino rats of the Sprague-Dawley strain obtained from the Sprague-Dawley Company served as subjects. The rats were 85-100 days old at the start of training. Throughout the experiment, they were individually housed under 12-h-light $(0800-2000 \mathrm{~h}), 12$-h-dark $(2000-0800 \mathrm{~h})$ light-dark cycle and were maintained on food and water ad lib. The subjects were randomly assigned to one of four independent groups $(n=8)$ defined by the training-testing interval (TTI) used $(.05,1,6$, and $24 \mathrm{~h})$. All subjects were handled 5 min each day for 1 week prior to the start of training.

Apparatus. The apparatus consisted of a manually operated T-maze with arms $10 \mathrm{~cm}$ wide $\times 60 \mathrm{~cm}$ long $\times 14 \mathrm{~cm}$ high, goalboxes $10 \mathrm{~cm}$ wide $\times 30 \mathrm{~cm}$ long $\times 14 \mathrm{~cm}$ high, and stem (startbox) $14 \mathrm{~cm}$ wide $\times 45 \mathrm{~cm}$ long $\times 14 \mathrm{~cm}$ high. The apparatus was constructed of wood and painted flat gray. All sections of the apparatus were covered by hinged hardware cloth tops and were separated by guillotine doors. Each goalbox contained an aluminum lick tube inserted through an angled hole in the distal end. The lick tube was connected to a $20-\mathrm{ml}$ plastic water bottle filled with a sugared milk solution (10\% sugar by weight). The subject's progress in the arm of the T-maze was measured by a .01-sec latency timer. The timer, which recorded committed latency, was activated by a photocell located $14 \mathrm{~cm}$ into the arm and was stopped by a photocell located $7 \mathrm{~cm}$ into the goalbox.

Procedure. The experiment proper consisted of three phases: pretraining, training, and retention testing. Four days prior to initiation of pretraining, the subjects were allowed access to the 
sugared milk solution in the home cage for a period of $2 \mathrm{~h} / \mathrm{day}$. Pretraining lasted 3 days and consisted of two 5-min reinforced placements in each goalbox each day. The training phase lasted 5 days and consisted of 4 trials/day on a position discrimination. On each day of training, all subjects received two forcedchoice trials to $\mathrm{S}+$ and two forced-choice trials to $\mathrm{S}-$. The forcedchoice procedure was used to insure equal exposure to $S+$ and $\mathrm{S}-$. The sequences of trials on the 5 days of training were as follows: Day 1, S+S-S+S-; Day $2 \mathrm{~S}+\mathrm{S}-\mathrm{S}-\mathrm{S}+$; Day $3, \mathrm{~S}-\mathrm{S}+\mathrm{S}+\mathrm{S}-$; Day 4, S-S+S-S+; Day 5, S-S-S+S+. During both training and testing, reinforcement on $S+$ trials consisted of $30 \mathrm{sec}$ access to the sugared milk solution in the goalbox and nonreinforcement on S- trials consisted of $30 \mathrm{sec}$ confinement in the unbaited goalbox. The retention test phase consisted of 16 free-choice trials on a reversal of the training discrimination $.05,1,6$, or $24 \mathrm{~h}$ following the last training trial. The use of a reversal of the original discrimination task in the testing phase has been shown to be a sensitive measure of retention of the original discrimination learning (e.g., Bryan \& Spear, 1976; Tribhowan et al., 1971). Poor retention of the original discrimination would be expected to result in less interference with learning of the new (reversal) task than would good retention of the original task. Thus, better performance on the reversal test would indicate poorer retention of the original discrimination.

The intertrial interval (ITI) in both training and testing phases was 10-15 sec. The experiment was run in four identical replications, with two subjects in each group in each replication (i.e., eight subjects per replication). The positions of S+ and Swere counterbalanced across replications. Committed latencies (i.e., the response time measured from the subject's initial choice of an arm of the maze until it entered the goalbox) were recorded in both training and testing. In addition, the percentage of incorrect choices in the retention test phase was also calculated for each subject.

\section{Results and Discussion}

Training. The data from the discrimination training phase in terms of latencies to S+ and S- may be seen in Figure 1. It appears that all four TTI conditions $(.05,1,6$, and $24 \mathrm{~h})$ learned the discrimination. That is, the differences in S+ and S- latencies seem to increase across training days for each TTI condition. These

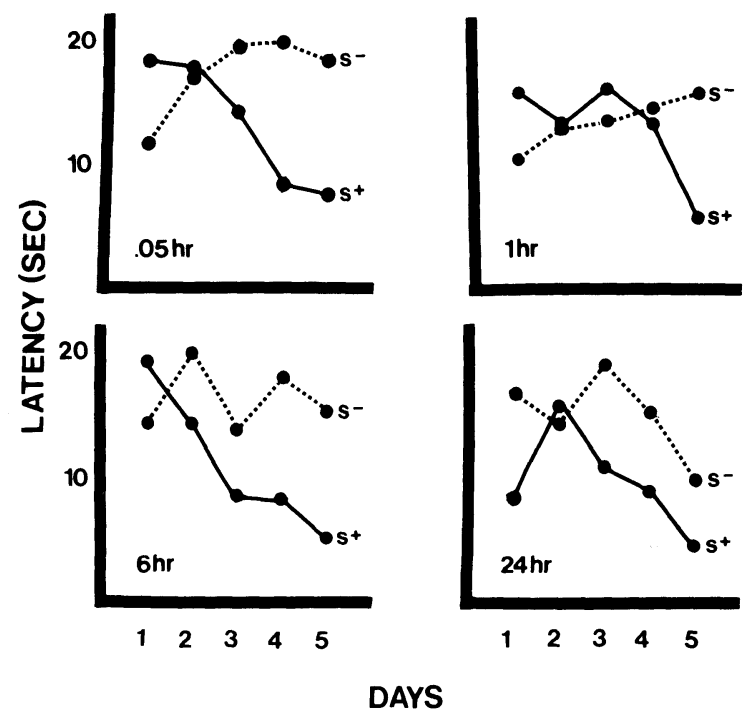

Figure 1. Discrimination training data (response latencies to $S+$ and $S_{-}$) from the four TTI groups. impressions were confirmed by the results of the 4 (TTI) by 2 (discriminanda) by 5 (days of training) factorial analysis of variance conducted on the data. Specifically, the main effect for the discriminanda was significant $[F(1,14)=8.73, p<.025]$, indicating shorter latencies to $\mathrm{S}+$ and longer latencies to $\mathrm{S}-$ during training. In addition, the interactions of Discriminanda by Days $[F(4,56)=44.75, p<.001]$ and TTI by Discriminanda by Days $[F(12,56)=9.59, p<.001]$ were also significant. The significant three-way interaction was further evaluated using Newman-Keuls post hoc comparison techniques $($ alpha $=.05)$. This analysis indicated that the latencies to S+ were significantly shorter than latencies to S- on Days 4 and 5 for the .05-h TTI condition, on Day 5 for the 1-h TTI condition, on Days 2, 4, and 5 for the 6-h TTI condition, and on Days 3, 4, and 5 for the 24-h TTI condition. Thus, it can be seen that all four TTI conditions learned the discrimination during the 5 days of training, although the differences in latencies to $S+$ and $S$ - appeared at different points in training for the TTI groups.

Testing. Mean percent errors on the retention test trials (discrimination reversal) for the TTI groups are depicted in Figure 2. As can be seen in the figure, retention performance seems to be poorer immediately after and $24 \mathrm{~h}$ after training than following intermediate retention intervals $(1$ and $6 \mathrm{~h})$. Thus, in the present experiment, retention appears to have been a U-shaped function of TTI. These observations were confirmed by the results of the one-way analysis of variance conducted on the percent errors data, which indicated a significant TTI effect $[F(3,28)=30.00, p<.001]$. The data were examined further using trend analysis procedures that revealed nonsignificant linear and cubic components $(\mathrm{Fs}<1)$ and a significant quadratic trend component $[\mathrm{F}(1,28)=$ $87.60, \mathrm{p}<.001]$. Thus, these data indicate that retention of an appetitive discrimination is a U-shaped function of TTI (i.e., a Kamin effect was observed).

The poorer performance in the discrimination reversal testing phase evidenced by the .05 - and 24-h groups (as a function of better retention of the original discrimination) in terms of percent errors might also be reflected in differences in response latency to $\mathrm{S}+$ and $\mathrm{S}-$. Namely, we might expect larger differences in S+ and S-latencies for the 1- and 6-h groups than for the .05- and 24-h groups, since the former demonstrated better performance during testing (in terms of percent errors) than did the latter. Examination of the latencies (shown in Table 1) for the four groups appears to confirm this suggestion. These data were analyzed via a 4 (TTI) by 2 (discriminanda) factorial analysis of variance. The results of the analysis revealed a marginally significant effect of TTI $[F(3,28)=2.82, p<.06]$, a significant effect of the discriminanda $[\mathrm{F}(1,28)=8.63$, $\mathrm{p}<.01$ ] , and a significant TTI by Discriminanda interaction $[F(3,28)=3.08, p<.05]$. Further evaluation of this interaction using the Newman-Keuls post hoc procedure (alpha $=.05)$ indicated that the $\mathrm{S}+$ and $\mathrm{S}-$ 


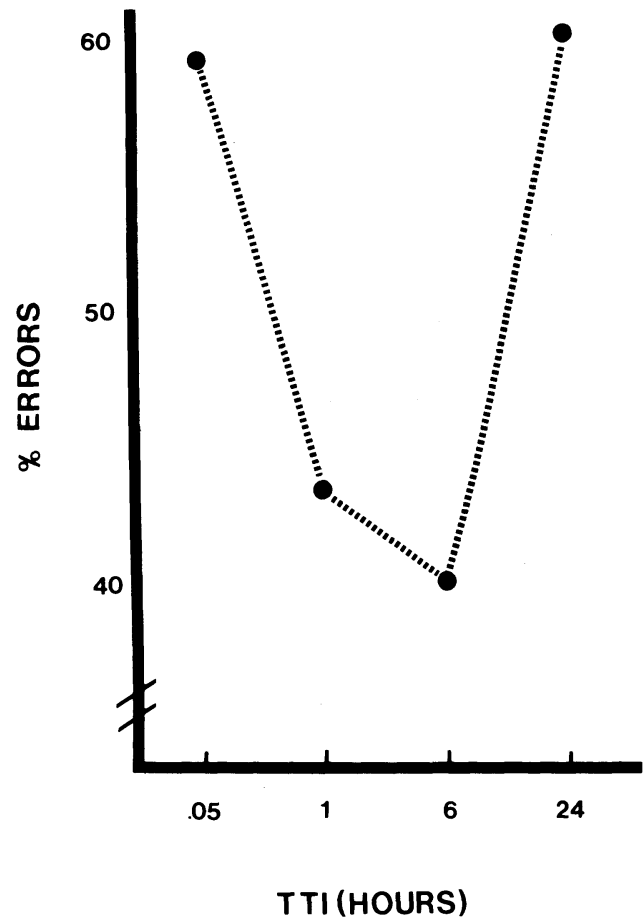

Figure 2. Mean percent errors on the retention test (reversal of the training discrimination) as a function of TTI.

Table 1

Mean Committed Latencies (in Seconds) in the Reinforced Arm (S+) and the Nonreinforced Arm (S-) of the T-Maze for All Groups in Experiment 1

\begin{tabular}{lcccc}
\hline & \multicolumn{4}{c}{ TTI } \\
\cline { 2 - 5 } & $.05 \mathrm{~h}$ & $1 \mathrm{~h}$ & $6 \mathrm{~h}$ & $24 \mathrm{~h}$ \\
\hline S+ Latency & 10.8 & 10.0 & 10.9 & 8.5 \\
S- Latency & 10.6 & 14.6 & 14.9 & 9.6 \\
\hline
\end{tabular}

latencies were significantly different in the 1- and 6-h TTI conditions and that the differences between $\mathrm{S}+$ and S- latencies in the .05- and 24-h TTI conditions were not significant. Thus, the latency data, which indicate better discrimination performance on the reversal test (and therefore poorer retention of original discrimination learning) for the 1- and 6-h TTI groups, confirm the conclusions drawn from the percent error data. Specifically, the function that represents retention of an appetitive discrimination response is approximately U-shaped (i.e., a Kamin effect).

\section{EXPERIMENT 2}

Although the data from Experiment 1 seem clear in indicating that retention of appetitive discrimination training is a U-shaped function of TTI, it is possible that this finding is an artifact of the procedure used. Specifically, it is possible that the transition from 4 forcedchoice trials per day in training to a single session of
16 free-choice trials in testing somehow interacted with the TTI used. Further, there may be some aspect of the single 16-trial testing session that produced the observed retention function. Experiment 2 was performed to investigate these possibilities. Specifically, the training phase consisted solely of goalbox placements (two to S+ and two to $S-$ per day for 5 days), followed by a single 16-trial retention test phase. If some aspect of the single session of retention testing produced the U-shaped retention function, as suggested above, then a U-shaped Kamin effect should also be observed in Experiment 2. However, if the Kamin effect observed in Experiment 1 actually reflects retention of appetitive discrimination training, as we believe, then a monotonic "retention" function should be observed in the present experiment.

\section{Method}

Subjects. The subjects were 32 experimentally naive male albino rats exactly like those used in Experiment 1 . Housing, maintenance, and handling were identical to those of Experiment 1 . The subjects were randomly assigned to one of four independent groups $(n=8)$ defined by the TTI used $(.05,1$, 6 , and $24 \mathrm{~h}$ ).

Apparatus. The apparatus was the same as that used in Experiment 1.

Procedure. The experiment proper consisted of three phases: pretraining, training, and retention testing. Pretraining was identical to that in Experiment 1. Training was similar to Experiment 1 , except that goalbox placements were used rather than forced-choice training trials. On each of the 5 days of training, each subject received two placements in the S+ goalbox and two placements in the $S$ - goalbox. The sequences of goalbox placements were identical to the forced-choice trial sequences in Experiment 1. Reinforcement, nonreinforcement, TTI, replications, counterbalancing, and the retention test phase were all identical to those of Experiment 1.

\section{Results and Discussion}

The data from the retention test phase revealed no differences between the groups in terms of percent errors on the discrimination reversal. Specifically, the mean percent errors were: $50.12 \%, 48.50 \%, 48.62 \%$, and $48.50 \%$ for the $.05-, 1-, 6-$, and $24-h$ groups, respectively. An analysis of variance on these data confirmed the lack of differences between the groups $(F<1)$. This absence of any differences between the TTI groups suggests that the Kamin effect observed in Experiment 1 was not an artifact of the procedure used. It should be noted, however, that the groups were performing only at a chance level (at least as reflected by percent errors) over the 16trial test phase. It is possible that, if a more extended discrimination test phase were used, differential discrimination performance might develop. There are two reasons to discount this possibility. First, 16 trials were sufficient for development of a discrimination in Experiment 1, at least for the 1- and 6-h groups. Second, an examination of the latency data for the present experiment (shown in Table 2) suggests that differential performance to $S+$ and S- (i.e., discrimination learning) occurred equally for all four TTI groups. This observation was confirmed by a 4 (TTI) by 2 (discriminanda) factorial analysis of variance conducted on these data. The results of the analysis 
Table 2

Mean Committed Latencies (in Seconds) in the Reinforced Arm (S+) and the Nonreinforced Arm (S-) of the T-Maze for All Groups in Experiment 2

\begin{tabular}{lcccc}
\hline & \multicolumn{4}{c}{ TTI } \\
\cline { 2 - 5 } & $.05 \mathrm{~h}$ & $1 \mathrm{~h}$ & $6 \mathrm{~h}$ & $24 \mathrm{~h}$ \\
\hline S+ Latency & 24.5 & 22.0 & 24.7 & 24.1 \\
S- Latency & 29.1 & 27.9 & 28.9 & 29.2 \\
\hline
\end{tabular}

revealed a nonsignificant effect for TTI $(F<1)$, a significant discriminanda effect $[F(1,28)=5.36, \mathrm{p}<.05]$, and a nonsignificant TTI by Discriminanda interaction $(F<1)$. Subsequent evaluation of the significant discriminanda main effect (Newman-Keuls comparisons at the .05 level) indicated better performance (i.e., shorter latencies) in S+ than in S- for all four TTI conditions. This finding indicates that discrimination learning was beginning to take place and that such learning was equal for all four groups. Presumably, the percent errors dependent measure would have reflected this learning had the test phase been extended. In any case, observation of equal discrimination learning (in terms of $\mathrm{S}+$ and $\mathrm{S}-$ latencies) in all four groups strongly suggests that a more extended test phase would not result in development of differential discrimination performance between the TTI groups.

The results of Experiment 2 clearly indicate that the Kamin effect observed in Experiment 1 was not an artifact of the procedure used. In addition, the present findings indicate that the Kamin effect observed in Experiment 1 was not due to other nonassociative effects arising from the experimental procedure. Thus the data from Experiment 2 support those from the first experiment indicating that the retention function for an appetitive discrimination task is U-shaped.

\section{GENERAL DISCUSSION}

The major finding of the present experiments was that retention of an appetitive discriminative response demonstrates the U-shaped Kamin effect that has been shown to be characteristic of several other learned responses in various paradigms under conditions of both appetitive and aversive motivation. Importantly, this result was obtained for dependent measures that are indexes of both learning (percent errors) and performance (response latencies).

Previous studies of retention of avoidance discrimination (e.g., Barrett, Leith, \& Ray, 1971) found Kamin effects in terms of performance variables (number of avoidances) but not in terms of variables reflecting retention of the avoidance learning (correct avoidances). Those investigators held that their findings of a Kamin effect only for performance measures indicated an underlying nonassociative mechanism. However, the results of the present appetitive retention experiments, wherein Kamin effects were observed for both performance and learning measures, seriously question those earlier findings. The present findings are supported as well by those of Bryan and Spear (1976), who observed Kamin effects for both performance and learning measures in retention of discriminated escape training. Both the present findings and those of Bryan and Spear strongly suggest an associative underlying mechanism for Kamin effect retention performance. Presumably, the discrepancies between the present findings and those reported by Barrett and his colleagues can be attributed to procedural differences, as has been mentioned elsewhere (Bryan \& Spear, 1976).

The present findings join a growing body of appetitive retention data (Seybert et al., 1979; Tribhowan et al., 1971) in supporting an associative account of nonmonotonic retention effects. As noted earlier, the nonassociative hypotheses (e.g., Barrett et al., 1971; Pinel \& Mucha, 1973) posit that Kamin effects are due to differential levels of motivation or activity resulting from the animals' experience with or exposure to an aversive stimulus (like shock). There are several reasons that indicate that such an explanation is untenable for the present data. First, no shock or other aversive stimuli were present in these experiments, and it would thus seem that the nonassociative hypotheses could not account for our results on that basis. Second, the present results were obtained from procedures involving no deprivation of any kind, and thus it is unlikely that the data presented here can be attributed to some nonassociative process resulting from a deprivation-induced motivational confound (as was possible in the Jaffard et al., 1974, and Tribhowan et al., 1971, studies). Finally, the results of Experiment 2 rule out a number of nonassociative procedural confounds. Thus, it would seem that the present findings cannot be accounted for by any of a number of possible nonassociative explanations. On the contrary, these results argue convincingly for an associative underlying mechanism, both for the present data and for those from studies investigating retention of aversively motivated responses.

\section{REFERENCES}

Anisman, H. Time-dependent variations in aversively motivated behaviors: Nonassociative effects of cholinergic and catecholaminergic activity. Psychological Review, 1975, 82, 359-385.

Barrett, R. J., Leith, N. J., \& RAY, O. S. Kamin effect in rats: Index of memory or shock-induced inhibition? Journal of Comparative and Physiological Psychology, 1971, 77, 234-239.

Bryan, R. C., \& SpEar, N. E. Forgetting of a discrimination after intervals of intermediate length: The Kamin effect with choice behavior. Journal of Experimental Psychology: Animal Behavior Processes, 1976, 2, 221-234.

Jaffard, R., Destrade, C., Soumireu-Mourat, B., \& Cardo, B. Time dependent improvement of performance on appetitive tasks in mice. Behavioral Biology, 1974, 11, 89-100.

KAMIN, L. J. Retention of an incompletely learned avoidance response. Journal of Comparative and Physiological Psychology, 1957, 50, 457-460.

Pinel, J. P. J., \& Mucha, R. F. Activity and reactivity in rats at various intervals after footshock. Canadian Journal of Psychology, 1973, 27, 112-118.

Seybert, J. A., Vandenberg, G. L., Harvey, R. J., Budd, J. R., \& McClanahan, L. G. Retention of appetitive instrumental behavior: The Kamin effect. Behavioral and Neural Biology, 1979, 26, 266-286.

Tribhowan, T., Rucker, W. B., \& McDiarmid, C. G. Demonstration of a Kamin-like effect after appetitive training. Psychonomic Science, 1971, 23, 41-42.

(Received for publication September 5, 1981.) 